TRANSACTIONS OF THE

AMERICAN MATHEMATICAL SOCIETY

Volume 349, Number 11, November 1997, Pages 4537-4554

S 0002-9947(97)02059-X

\title{
COMPACT GROUPS AND FIXED POINT SETS
}

\author{
ALEX CHIGOGIDZE, KARL H. HOFMANN, AND JOHN R. MARTIN
}

\begin{abstract}
Some structure theorems for compact abelian groups are derived and used to show that every closed subset of an infinite compact metrizable group is the fixed point set of an autohomeomorphism. It is also shown that any metrizable product containing a positive-dimensional compact group as a factor has the property that every closed subset is the fixed point set of an autohomeomorphism.
\end{abstract}

\section{INTRODUCTION}

A space $X$ is defined to have the complete invariance property (CIP) if every nonempty closed subset of $X$ is the fixed point set of a (continuous) self-mapping of $X$ [23]. If this condition holds for autohomeomorphisms of $X$, then we say that $X$ has the complete invariance property with respect to homeomorphisms (CIPH) [13]. A survey of results concerning CIP for metric spaces may be found in [20], and a number of nonmetric results may be found in [16]. Some spaces known to have CIPH are even-dimensional Euclidean balls [18], compact surfaces and positivedimensional spheres [19], Menger manifolds [12], the Hilbert cube and metrizable product spaces which have the real line or an odd-dimensional sphere as a factor [13]. Metrizable topological groups are known to have CIP if they are locally compact or contain an arc [15]. In [16] it is shown that an uncountable self-product of circles, real lines or two-point spaces has CIP and that connected subgroups of the plane and compact groups need not have CIP. To best of the authors' knowledge this is the first investigation devoted to an explicit statement that topological groups in a certain class have CIPH.

In this paper a general structure theorem for compact abelian groups is proved, and this is used as a basis for a self-contained development yielding an explicit structure theorem for finite-dimensional compact abelian groups. These results are used to show that a nondegenerate compact metrizable group has CIPH iff it is infinite. It is also shown that the product of two metrizable spaces has CIPH if one of the factors is a positive-dimensional compact group. In particular, every proper solenoid is an indecomposable continuum whose product with any metrizable space has CIPH. Furthermore, if other examples of nondegenerate indecomposable continua possessing CIPH exist, they cannot admit topological group structures since we show that a nondegenerate compact group is an indecomposable continuum iff it is a proper solenoid. The paper concludes with some results which show

Received by the editors December 15, 1995.

1991 Mathematics Subject Classification. Primary 22C05, 54H25; Secondary 22D35.

Key words and phrases. Compact group, character group, flow, fixed point set.

The first named author was partially supported by an NSERC research grant.

(c)1997 American Mathematical Society 
that conditions which ensure CIPH for metrizable spaces may fail to do so for nonmetrizable spaces. For instance, it is shown that the product space of a compact connected Lie group with a product of closed unit intervals (or two-point discrete groups) has CIPH iff the product space is metrizable.

\section{Structure Theorems for Compact Abelian Groups}

We assume as known the duality between the category of compact abelian groups and that of discrete abelian groups according to Pontryagin and van Kampen. For two topological abelian groups $A$ and $B$ we shall always denote by $\operatorname{Hom}(A, B)$ the topological abelian group of all continuous group homomorphisms from $A$ to $B$ equipped with the compact open topology. We use the notation $\mathbb{R}$ for the locally compact additive group of reals and $\mathbb{T}=\mathbb{R} / \mathbb{Z}$ for the circle group. We write $\widehat{G}=\operatorname{Hom}(G, \mathbb{T})$ for the group of characters $\chi: G \rightarrow \mathbb{T}$ (continuous in the category of compact abelian groups). The duality theorem states that the evaluation morphism $\eta_{G}: G \rightarrow \hat{\hat{G}}, \eta_{G}(g)(\chi)=\chi(g)$ is an isomorphism in all cases. One identifies $\widehat{\mathbb{R}}$ with $\mathbb{R}$ in a natural way.

Definition 2.1. For a compact topological group $G$ we write

$$
L(G) \stackrel{\text { def }}{=} \operatorname{Hom}(\mathbb{R}, G) \cong \operatorname{Hom}(\widehat{G}, \mathbb{R})
$$

and note that $L(G)$ is a real topological vector space with the topology of uniform convergence on compact sets. We set $\exp : L(G) \rightarrow G$, $\exp X=X(1)$.

All one-parameter subgroups of $G$ are of the form $X=(r \mapsto \exp r \cdot X): \mathbb{R} \rightarrow$ $G$. We note that $\operatorname{Hom}(\widehat{G}, \mathbb{R}) \cong \operatorname{Hom}(\mathbb{R} \otimes \widehat{G}, \mathbb{R})$ is the vector space of all linear functionals on the real vector space $\mathbb{R} \otimes \widehat{G}$, i.e., the algebraic dual $(\mathbb{R} \otimes \widehat{G})^{*}$ given the weak-* topology. The only isomorphy invariant is the cardinal

$$
d \stackrel{\text { def }}{=} \operatorname{dim}_{\mathbb{R}}(\mathbb{R} \otimes \widehat{G})=\operatorname{dim}_{\mathbb{Q}}(\mathbb{Q} \otimes \widehat{G})=\operatorname{rank} \widehat{G} .
$$

Therefore, $L(G)$ is algebraically and topologically isomorphic to $\mathbb{R}^{d}$.

For compact Hausdorff spaces, the terms zero-dimensional and totally disconnected are synonymous. (This is sometimes referred to as Vedenissoff's Theorem [22].) Below $|A|$ stands for the cardinality of a set $A$, and $\omega$ denotes the first infinite cardinal number.

Proposition 2.2. For a compact abelian group $G$ there is a compact zero-dimensional subgroup $\Delta$ such that the homomorphism

$$
\phi: \Delta \times L(G) \rightarrow G, \quad \phi(d, X)=d \exp X
$$

satisfies the following conditions:

(i) $\phi$ is continuous, surjective and open, i.e., is a quotient morphism.

(ii) $\operatorname{ker} \phi$ is algebraically and topologically isomorphic to $D \stackrel{\text { def }}{=} \exp ^{-1}(\Delta)$, and $D$ is a closed totally disconnected subgroup of $L(G)$. In particular, it does not contain any nonzero vector spaces.

(iii) $\phi(\{1\} \times L(G))=\exp L(G)$ is dense in $G_{0}$, the identity component of $G$.

Proof. By Zorn's Lemma, the abelian group $\widehat{G}$ contains a free subgroup $F$ of maximal rank. Then $E \stackrel{\text { def }}{=} \widehat{G} / F$ is a torsion group. We set $\Delta=F^{\perp}$ (the annihilator of $F$ in $G$ ). Then, by duality, $\widehat{\Delta} \cong E$. Since $E$ is a torsion group, $\Delta$ is a totally disconnected subgroup. Clearly, $\phi: \Delta \times L(G) \rightarrow G$ defined by $\phi(d, X)=d \exp X=d X(1)$ 
is a well defined continuous homomorphism. Further, $T \stackrel{\text { def }}{=} \widehat{F}$, as the character group of a direct sum of copies of $\mathbb{Z}$, is a product of copies of $\mathbb{T} \cong \widehat{\mathbb{Z}}$, i.e., a torus. In particular, exp: $L(T) \rightarrow T$ is surjective. Let $p: G \rightarrow T$ denote the quotient morphism which identifies $G / \Delta$ with $T$. The morphism

$$
L(p)=\operatorname{Hom}(\mathbb{R}, p): L(G)=\operatorname{Hom}(\mathbb{R}, G) \rightarrow \operatorname{Hom}(\mathbb{R}, T)=L(T)
$$

is an isomorphism since $\operatorname{Hom}(\operatorname{incl}, \mathbb{R}): \operatorname{Hom}(\widehat{G}, \mathbb{R}) \rightarrow \operatorname{Hom}(F, \mathbb{R})$ is an isomorphism $($ as $\operatorname{Hom}(E, \mathbb{R})=\{0\})$.

Proof of (i). The surjectivity of $\phi$. The exact sequence

$$
0 \rightarrow F \stackrel{\text { incl }}{\longrightarrow} \widehat{G} \stackrel{\text { quot }}{\longrightarrow} E \rightarrow 0
$$

gives an exact sequence of compact groups

$$
0 \rightarrow \Delta \stackrel{\text { incl }}{\longrightarrow} G \stackrel{p}{\longrightarrow} T \rightarrow 0 \text {. }
$$

Since

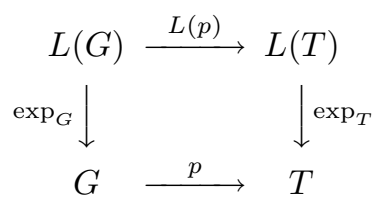

is commutative, $p\left(\exp _{G} L(G)\right)=T$. Hence $\Delta \exp L(G)=(\operatorname{ker} p) \exp L(G)=G$. Thus $\phi$ is surjective.

The openness of $\phi$. For every subgroup $F^{\prime}$ of $G$ containing $F$ such that $F^{\prime} / F$ is finite, we get exact sequences

$$
0 \rightarrow F^{\prime} \stackrel{\text { incl }}{\longrightarrow} \widehat{G} \stackrel{\text { quot }}{\longrightarrow} E^{\prime} \rightarrow 0
$$

and

$$
0 \rightarrow \Delta^{\prime} \stackrel{\text { incl }}{\longrightarrow} G \stackrel{p^{\prime}}{\rightarrow} T^{\prime} \rightarrow 0
$$

and $\Delta^{\prime}$ has finite index in $\Delta$. We note that

- the family of all $\Delta^{\prime}$ intersects in $\{1\}$,

- the argument showing $\Delta \exp L(G)=G$ above shows in exactly the same fashion that $\Delta^{\prime} \exp L(G)=G$.

Since $F / F^{\prime}$ is finite, we can write $F=F_{1} \oplus F_{2}$ such that $F^{\prime}=F_{1} \oplus F_{2}^{\prime}$ with $F_{2} / F_{2}^{\prime} \cong F / F^{\prime}$.

Accordingly, we have $T=T_{1} \times T_{2}, T^{\prime}=T_{1} \times T_{2}^{\prime}$ (with a natural identification), $\operatorname{Hom}(F, \mathbb{R})=\operatorname{Hom}\left(F_{1}, \mathbb{R}\right) \oplus \operatorname{Hom}\left(F_{2}, \mathbb{R}\right)$, and thus $L(T)=L(T)_{1} \oplus L(T)_{2}$. Correspondingly, $L(G)=L(G)_{1} \oplus L(G)_{2}$, where $L(G)_{j}=L(p)^{-1}\left(L\left(T_{j}\right)\right), j=1,2$. We write

$$
0 \rightarrow \Delta^{\prime} \stackrel{\text { incl }}{\rightarrow} G \stackrel{p^{\prime}}{\rightarrow} T_{1} \oplus T_{2}^{\prime} \rightarrow 0
$$

and note that $L(G)_{1}=L\left(p^{\prime}\right)^{-1}\left(L\left(T_{1}\right)\right)$. Suppose that $p^{\prime}(g) \in T_{1}$. Then there is an $X_{1} \in L(G)_{1}$ such that $\exp _{T_{1}} L\left(p^{\prime}\right)\left(X_{1}\right)=p^{\prime}\left(\exp _{G} X_{1}\right)=p^{\prime}(g)$. Thus $g \exp _{G}\left(-X_{1}\right)$ $\in \operatorname{ker} p^{\prime}=\Delta^{\prime}$. Therefore, $\Delta^{\prime} \exp _{G} L(G)_{1}=\left(p^{\prime}\right)^{-1}\left(T_{1}\right)$. Hence $\Delta^{\prime} \exp _{G} L(G)_{1}$ is closed in $G$.

Let $U \times V$ be a product zero-neighborhood of $\Delta \times L(G)$. Then $U$ contains a subgroup $\Delta^{\prime}$ of $\Delta$ which is open in $\Delta$ and has finite index in $\Delta$, and $V$ contains a vector subspace $L(G)_{1}$ of $L(G)$ with $\operatorname{dim} L(G) / L(G)_{1}<\infty$. 
It is no loss of generality to assume that $U=U \Delta^{\prime}$ and $V=V L(G)_{1}$, i.e., that $U$ and $V$ are "saturated". We observe that

$$
(\Delta \times L(G)) /\left(\Delta_{1} \times L(G)_{1}\right) \cong \Delta / \Delta_{1} \times L(G) / L(G)_{1}
$$

is a Lie group with finitely many components, and $\phi$ induces a surjective homomorphism of Lie groups $\phi^{\prime}:(\Delta \times L(G)) /\left(\Delta_{1} \times L(G)_{1}\right) \rightarrow G / \Delta^{\prime} \exp L(G)_{1}$. By the Open Mapping Theorem for locally compact groups, $\phi^{\prime}$ is open. The following diagram is commutative:

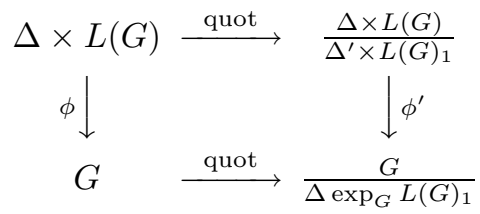

Since $U \times V$ is saturated, we may conclude that $\phi(U \times V)$ is open in $G$.

Proof of (ii). We have $\operatorname{ker} \phi=\{(d, X) \in \Delta \times L(G): d \exp X=1\}$. The map $X \mapsto(\exp -X, X): D \rightarrow \operatorname{ker} \phi$ is therefore bijective. This morphism has the inverse $(d, X) \mapsto X: \operatorname{ker} \phi \rightarrow D$. Hence it is an algebraic and topological isomorphism. The projection $\operatorname{pr}_{\Delta}: \Delta \times L(G) \rightarrow \Delta$ induces an injective morphism $j: \operatorname{ker} \phi \rightarrow \Delta$. Let $C \stackrel{\text { def }}{=}(\operatorname{ker} \phi)_{0}$ denote the identity component of $\operatorname{ker} \phi$. Then $j(C)$ is a connected subgroup of the totally disconnected group $\Delta$. It is therefore singleton and thus $C$ is singleton, i.e., $\operatorname{ker} \phi$ and thus $D$ are totally disconnected.

Proof of (iii). This statement is known. Here is a short argument: Set $H=$ $\overline{\exp L(G)}$. The inclusion $j: H \rightarrow G$ induces an isomorphism $L(j): L(H) \rightarrow L(G)$ in view of the definitions. Since $L(\cdot)=\operatorname{Hom}(\mathbb{R}, \cdot)$, the following sequence is exact:

$$
0 \rightarrow L(H) \stackrel{L(j)}{\rightarrow} L(G) \rightarrow L(G / H) \rightarrow \operatorname{Ext}(\mathbb{R}, H) .
$$

But $H$ is divisible as the underlying group of a connected compact abelian group. Hence $H$ is injective, and thus $\operatorname{Ext}(\mathbb{R}, H)=\{0\}$. Hence $L(G / H)=\{0\}$. Since every nondegenerate compact connected abelian group contains a nondegenerate one-parameter subgroup, $(G / H)_{0}=\{0\}$. Therefore $H=G_{0}$.

To the best of our knowledge Proposition 2.2 is new in the generality stated here. It would be more valuable if we knew the totally disconnected closed subgroups of a topological vector space $\mathbb{R}^{d}$ for any cardinal $d$. In finite dimensions, this matter is no problem at all, as we shall record in the following.

Proposition 2.3 (Dixmier, see [8]). For any compact abelian group $G$ the subgroup $\exp L(G)$ is exactly the arc component of 0 in $G$.

We can write $\pi_{0}(G)=G / \exp L(G)$, and Dixmier has shown that $\pi_{0}(G) \cong$ $\operatorname{Ext}(\widehat{G}, \mathbb{Z})$.

Corollary 2.4. The morphism $\phi: \Delta \times L(G) \rightarrow G$ maps arc components onto arc components, and $\pi_{0}(G) \cong \frac{\Delta}{\Delta \exp L(G)}$ (algebraically).

Theorem 2.5. The following conditions are equivalent for a compact abelian group $G$ and a natural number $n$ :

1. $\operatorname{rank} \widehat{G}=\operatorname{dim}_{\mathbb{Q}}(\mathbb{Q} \otimes \widehat{G})=n$. 
2. There is an exact sequence

$$
0 \rightarrow \operatorname{tor}(\widehat{G}) \rightarrow \widehat{G} \rightarrow \mathbb{Q}^{n} \rightarrow E \rightarrow 0
$$

with the torsion subgroup $\operatorname{tor}(\widehat{G})$ and some torsion group $E$.

3. There is an exact sequence

$$
0 \rightarrow \mathbb{Z}^{n} \rightarrow \widehat{G} \rightarrow E \rightarrow 0
$$

with some torsion group $E$.

4. There is a compact zero-dimensional subgroup $Z$ of $(\widehat{\mathbb{Q}})^{n}$ and an exact sequence

$$
0 \rightarrow Z \rightarrow(\widehat{\mathbb{Q}})^{n} \rightarrow G \rightarrow G / G_{0} \rightarrow 0,
$$

where $G_{0}$ is the identity component of $G$.

5. There is a compact zero-dimensional subgroup $\Delta$ of $G$ and an exact sequence

$$
0 \rightarrow \Delta \rightarrow G \rightarrow \mathbb{T}^{n} \rightarrow 0
$$

6. $\operatorname{dim} L(G)=n$

7. There is a compact zero-dimensional subgroup $\Delta$ of $G$ and a quotient homomorphism $\phi: \Delta \times \mathbb{R}^{n} \rightarrow G$ which has a discrete kernel. In particular, $\phi$ yields a local isomorphism of $\Delta \times \mathbb{R}^{n}$ and $G$.

8. The identity of $G$ has a neighborhood basis each member of which is homeomorphic to $D \times C^{n}$ with some totally disconnected compact space $D$ and an $n$-cell $C^{n}$.

Proof. In the theory of abelian groups, conditions (1), (2), and (3) are all known to be equivalent to the statement that the (torsion free) rank of $\widehat{G}$ is $n$. By duality, (4) is equivalent to (2), and (5) is equivalent to (3).

Condition (6), saying $\operatorname{dim} \operatorname{Hom}(\mathbb{R}, G)=n$, in view of

$$
\operatorname{Hom}(\mathbb{R}, G) \cong \operatorname{Hom}(\widehat{G}, \mathbb{R}) \cong \operatorname{Hom}(\mathbb{Q} \otimes \widehat{G}, \mathbb{R}) \cong \mathbb{R}^{\operatorname{dim}_{\mathbb{Q}}(\mathbb{Q} \otimes \widehat{G})}
$$

is equivalent to (1).

$(1) \Longrightarrow(7)$. By (1) we have $L(G) \cong \mathbb{R}^{n}$. By Proposition 2.2 , we obtain $\phi: \Delta \times$ $\mathbb{R}^{n} \rightarrow G$ as asserted, because $\operatorname{ker} \phi \cong \exp ^{-1}(\Delta)$ and this closed subgroup of $L(G) \cong$ $\mathbb{R}^{n}$ does not contain vector subgroups, hence is discrete.

$(7) \Longrightarrow(8)$ is immediate.

$(8) \Longrightarrow(6)$. Let $U$ be an identity neighborhood of $G$ and $h: D \times C^{n} \rightarrow U$ a homeomorphism with a closed $n$-cell $C^{n}$.

We let $p: G \rightarrow T$ be as in the proof of Proposition 2.2 so that $L(p): L(G) \rightarrow L(T)$ is an isomorphism. Let $K=\operatorname{ker}\left(L(p) \exp _{T}\right)=\operatorname{ker}\left(p \exp _{G}\right)$ as before. We may assume that $\Delta=\operatorname{ker} p$ is such that there is a compact identity neighborhood $V \subseteq U$ with $V \Delta=V$.

We recall the free subgroup $F$ of $\widehat{G}$ and let $\left(\epsilon_{j}\right)_{j \in J}$ be a basis of $F$. Set $\rho_{0}: \mathbb{R}^{J} \rightarrow$ $\operatorname{Hom}(F, \mathbb{R}), \rho_{0}\left(\left(r_{j}\right)_{j \in J}\right)\left(\sum_{j \in J} r_{j} \cdot \epsilon_{j}\right)=\sum_{j \in J} \epsilon_{j}\left(r_{j}\right)$. Then $\rho_{0}$ is an isomorphism of topological groups, and $\operatorname{since} \operatorname{Hom}(F, \mathbb{R}) \cong \operatorname{Hom}(\mathbb{R}, T) \cong L(T)$ we have an isomorphism of topological groups $\rho: \mathbb{R}^{J} \rightarrow L(T)$. Moreover, if we write $F=$ $\bigoplus_{j \in J} \mathbb{Z} \cdot \epsilon_{j}$ and, accordingly, $T=\prod_{j \in J} \widehat{\mathbb{Z} \cdot \epsilon_{j}} \stackrel{\sigma}{\rightarrow} \mathbb{T}^{J}$, then $\exp ^{\prime}=\sigma \exp _{T} \rho: \mathbb{R}^{J} \rightarrow$ $\mathbb{T}^{J}$ has the kernel $\mathbb{Z}^{J}$. It follows that $K=\operatorname{ker} \exp _{T} L(p)$ maps isomorphically onto $\mathbb{Z}^{J}$ under $\rho L(p)$. Now $p(V)$ is an identity neighborhood of $T \cong \mathbb{T}^{J}$. Then we find an $\frac{1}{2}>r>0$ so that $S_{r}=L(p)^{-1} \rho^{-1}\left([-r, r]^{J}\right)$ satisfies $S_{2 r} \cap \mathbb{Z}^{J}=\{0\}$ and $p\left(\exp _{G} S_{r}\right)=\exp _{T} L(p)\left(S_{r}\right) \subseteq p(V)$. Then $p \exp _{G}$ maps $S_{r}$ homeomorphically 
into $T$, and so a fortiori $\exp _{G}$ maps $S_{r}$ homeomorphically into $G$. But $V=V \Delta=$ $p^{-1} p(V) \subseteq U$. Hence $\exp _{G} S_{r} \subseteq U$, and $e=\exp _{G} \mid S_{r}: S_{r} \rightarrow U$ is a homeomorphism onto the image. Then $h^{-1} e: S_{r} \rightarrow D \times C^{n}$ is a homeomorphism onto the image. If $(d, c)=h^{-1}(1)$, then $h\left(\{d\} \times C^{n}\right)$ is the connected component of 1 in $U$, and thus $h^{-1} e\left(S_{r}\right)$ is a homeomorphic copy of $S_{r}$ contained in the $n$-cell $\{d\} \times C^{n}$. But $S_{r}$ is homeomorphic to $[-r, r]^{J}$, hence to $[-1,1]^{J}$. Since $[-1,1]^{J}$ contains $[-1,1]^{m}$ for $m=0,1,2, \ldots,|J|$, this entails $|J| \leq n$ by the invariance of domain.

Thus $m \stackrel{\text { def }}{=} \operatorname{dim} L(G)=|J| \leq n$. Then by “(6) $\Rightarrow(8)$ ", there are arbitrarily small identity neighborhoods homeomorphic to $D^{\prime} \times C^{m}$ with a totally disconnected compact space $D^{\prime}$ and an $m$-cell $C^{m}$. Thus, by hypothesis (8), an $n$-cell must be contained in an $m$-cell, and this implies $n \leq m$ by invariance of domain.

Definition 2.6. Let $G$ be a compact abelian group. Then we set

$$
\operatorname{dim} G=\operatorname{dim}_{\mathbb{Q}}(\mathbb{Q} \otimes \widehat{G})
$$

and call this cardinal the dimension of $G$. If $\operatorname{dim} G$ is finite, then $G$ is called finite-dimensional, and otherwise infinite-dimensional.

Corollary 2.7. If for a compact abelian group $G$ there is a natural number $n$ such that the equivalent conditions of Theorem 2.5 are satisfied, then $n=\operatorname{dim} G$.

Suppose that DIM is a dimension function defined for compact topological spaces such that

- it assigns to a product $D \times C^{n}$ of a compact totally disconnected space and a compact $n$-cell $C^{n}$ the dimension $n$,

- it assigns to a homogeneous space containing such a subspace with nonempty interior the dimension $n$, and

- it assigns to a compact space the dimension $\infty$ if it contains a homeomorphic copy of $[-1,1]^{\omega}$.

Then we will have

$$
\operatorname{DIM}(G)= \begin{cases}\operatorname{dim} G & \text { if } G \text { is finite-dimensional, } \\ \infty & \text { if } G \text { is infinite-dimensional. }\end{cases}
$$

Proposition 2.8. (Pontryagin) For a compact abelian group $G$ one has $w(G)=$ $|\widehat{G}|$, where $w(G)$ denotes the weight of the space $G$.

From Theorem 2.5 for an infinite compact group we note that

$$
|\widehat{G} / \operatorname{tor}(\widehat{G})|=|\mathbb{Q} \otimes \widehat{G}|=\max \{\omega, \operatorname{dim} G\} .
$$

We derive

Proposition 2.9. For an infinite compact abelian group $G$,

$$
w(G)=w\left(G / G_{0}\right) \max \{\omega, \operatorname{dim} G\} .
$$

In particular, a finite-dimensional connected compact abelian group is metric.

More generally, a finite-dimensional compact abelian group is metrizable iff $w\left(G / G_{0}\right) \leq \omega$.

For the proof of the next proposition we need some facts on abelian groups.

Lemma 2.10 (Pontryagin). Let $A$ be a countable torsion free group and assume that every finite rank pure subgroup is free. Then $A$ is free. 
Proof. The claim is clear if $\operatorname{rank} A<\infty$. We now assume $\operatorname{rank} A=\omega$. Let $\left\{e_{1}, e_{2}, \ldots\right\}$ denote a maximal free set. Define $P_{j}$ to be the free pure subgroup generated by $\left\{e_{1}, \ldots, e_{j}\right\}$. Inductively define a free set $\left\{f_{1}, f_{2}, \ldots\right\}$ such that $f_{1}, \ldots, f_{j(n)}$ is a basis of $P_{n}$ for a suitable sequence of natural numbers $j(n)$. Indeed, if $f_{1}, \ldots, f_{j}(n)$ is a basis of $P_{n}$, then $P_{n+1}$ is free and the pure subgroup $P_{n}$ is a direct summand (in view of the elementary divisor theorem). Hence we can complement the basis of $P_{n}$ to a basis $f_{1}, \ldots, f_{j(n)}, f_{j(n)+1}, \ldots, f_{j(n+1)}$. This completes the recursion. The span of the free set $f_{1}, \ldots$ contains all $P_{n}$ and thus all $e_{n}$, and thus it is $A$.

Now let $A$ be an abelian group. Let $\mathcal{K}$ denote the set of all subgroups $K$ of $A$ such that $A / K$ is free. Then $\mathcal{K}$ is a filter basis; for if $K_{1}, K_{2} \in \mathcal{K}$ then $K=K_{1} \cap K_{2}$ is the kernel of the map $a \mapsto\left(a+K_{1}, a+K_{2}\right): A \rightarrow A / K_{1} \times A / K_{2}$. The image of this homomorphism is a subgroup of a free abelian group and is therefore a free abelian group. We can form

$$
K_{\infty}=K_{\infty}(A)=\bigcap \mathcal{K} .
$$

(Cf. [9].) Then all morphisms into free groups factor through $A \rightarrow A / K_{\infty}$, and the homomorphisms $A / K_{\infty} \rightarrow \mathbb{Z}$ separate the points. In particular, $A / K_{\infty}$ is torsion free and $\operatorname{tor}(A) \subseteq K_{\infty}$. Notably, $K_{\infty}$ is a pure subgroup.

For a subgroup $H$ of a torsion free abelian group $A$, the group

$$
[H] \stackrel{\text { def }}{=}\{a \in A: \exists n \in \mathbb{N} \text { such that } n \cdot a \in H\}
$$

is the smallest pure subgroup containing $H$. One also calls $A$ torsionless if $K_{\infty}(A)=$ $\{0\}$.

Lemma 2.11. If $K_{\infty}(A)=\{0\}$, i.e., if $A$ is torsionless, then every finite rank pure subgroup of $A$ is free.

Proof. Let $P$ be a finite rank pure subgroup of $A$ and $F$ a maximal rank free subgroup contained in $P$. Then $P=[F]$. Since $K_{\infty}(A)=\{0\}$, there is a subgroup $K \in \mathcal{K}$ such that $F \cap K=\{0\}$. It follows that $P \cap K=\{0\}$, for if $p \in P \cap K$ then there is an $m \in \mathbb{N}$ such that $m \cdot p \in F \cap K=\{0\}$, whence $p=0$ since $A$ is torsion free. The map $x \mapsto x+K: P \rightarrow A / K$ is therefore injective. But $A / K$ is free by the definition of $\mathcal{K}$, and thus $P$ is a free abelian group as a subgroup of a free abelian group, as we wanted to show.

Lemma 2.12 (Main Lemma). Let $A$ be an abelian group such that $A / K_{\infty}(A)$ is countable. Then $A$ contains a free subgroup $F$ such that $A=F \oplus K_{\infty}(A)$. Moreover, $K_{\infty}(A)$ does not have any nondegenerate free quotients.

Proof. The group $A / K_{\infty}(A)$ is torsion free and countable, and the morphisms into free groups separate the points. Hence $K_{\infty}\left(A / K_{\infty}(A)\right)=\{0\}$. Thus from Lemma 2.11 we know that every finite rank pure subgroup is free. Then, by Lemma 2.10, the quotient $A / K_{\infty}(A)$ is free. Since free groups are projective, this implies the existence of $F$ as asserted. Again any free quotient of $K_{\infty}(A)$ splits, so $K_{\infty}(A)=$ $F^{\prime} \oplus K$ with a free $F^{\prime}$ isomorphic to the free quotient. But then $F \oplus F^{\prime}$ is free and thus $K \in \mathcal{K}$. It follows that $K_{\infty}(A) \subseteq K$ and that, as a consequence, $F^{\prime}$ is degenerate. 


\section{Theorem 2.13.}

(i) If $T$ is a torus subgroup (a product of circle groups) of a compact abelian group $G$, then there is a (not necessarily unique) subgroup $C$ such that

$$
(t, c) \mapsto t c: T \times C \rightarrow G
$$

is an isomorphism of compact groups.

(ii) Every compact abelian group $G$ contains a (fully characteristic) unique smallest closed subgroup $M=M(G)$ containing all circle subgroups, and $M^{\perp}=$ $K_{\infty}(\widehat{G})$. The subgroup $M(G)$ is always locally connected.

(iii) If $M(G)$ satisfies the first (and hence the second) axiom of countability, i.e., is metric, then $M(G)$ is a torus and $G$ contains a closed subgroup $C$ not containing circle groups such that

$$
(m, c) \mapsto m c: M(G) \times C \rightarrow G
$$

is an isomorphism of compact groups, i.e., $G$ is a direct product of a metric torus and a torus free compact subgroup.

(iv) Every metric compact abelian group $G$ is the direct product of a fully characteristic maximal torus subgroup $M(G)$ and some torus free closed subgroup.

Proof. Part (i) just restates the fact, by duality, that in the category of abelian groups the free groups are the projectives and that a homomorphism onto a projective splits.

(ii) We let $M$ be the closure of the group generated by the union of all circle groups. This is the smallest closed subgroup of $G$ containing all circle groups and is, therefore, fully characteristic. The product of two tori is a torus (it is clearly a compact subgroup, and the first factor splits by (i); the complementary factor is a homomorphic image of the second factor and is, therefore, a torus). Hence the set of all tori in $G$ is upwards directed, and $M$ is the closure of its union. Dually, the annihilator of $M$ is the intersection of all $T^{\perp}$ as $T$ ranges through all tori. By duality, the $T^{\perp}$ are exactly the members of $\mathcal{K}(\widehat{G})$. Thus $M(G)^{\perp}=K_{\infty}(\widehat{G})$. In particular, $\widehat{M(G)} \cong \widehat{G} / K_{\infty}(\widehat{G})$. Thus by Lemma 2.11 , in the character group of $M(G)$ every finite rank pure subgroup is free. By a theorem of Pontryagin [17, II, Satz 48, Seite 33], this property characterizes locally connected compact abelian groups.

(iii) Now suppose that $M(G)$ is metric. Then $\widehat{G} / M(G)^{\perp} \cong \widehat{G}$ is countable. By (ii) we have $M(G)^{\perp}=K_{\infty}(\widehat{G})$. Now Lemma 2.12 implies $\widehat{G}=F \oplus M(G)^{\perp}$ with a (countable) free group $F$. We set $C=F^{\perp} \subseteq G$ and obtain $G \cong M(G) \times C$, as stated in (iii). Since $M(G)$ contains all circle groups, $C$ does not contain any circle groups. (Equivalently: $K_{\infty}(\widehat{G})$ does not have any free quotients by Lemma 2.12.)

(iv) If $G$ is metric, then, in particular, (iii) applies and proves the assertion.

It is known that, for metric compact connected groups, arc connectivity, local connectivity, and being a torus group are equivalent properties. The character group $G$ of $\mathbb{Z}^{\omega}$ is connected, locally connected, but not arcwise connected, let alone a torus group. But for this $G$ we have $G=M(G)$. This shows that metrizability in (iii) is essential.

Dixmier [8] has observed (using the Axiom of Choice) that there is an abelian group $A$ containing a subgroup $Z$ isomorphic to $\mathbb{Z}$ such that $A / Z \cong \mathbb{Z}^{\omega}$, and that $Z$ does not split. If $g \in Z$ is a generator and if there existed a homomorphism 
$f: A \rightarrow F$ into a free group $F$ with $f(g) \neq 0$, then $[f(Z)] \cong \mathbb{Z}$ and $F=F_{1} \oplus[f(Z)]$; hence there would exist a morphism $\phi: A \rightarrow \mathbb{Z}$ with $\phi(Z)=\mathbb{Z}$, yielding $A=H \oplus Z$ and thus contradicting the fact that $Z$ does not split. Hence $Z \subseteq K_{\infty}(A)$. Since $A / Z \cong \mathbb{Z}^{\omega}$, the free quotients separate the points of $A / Z$, whence $Z=K_{\infty}(A)$. One notes that every finitely generated pure subgroup of $A$ is free. As a consequence $G \stackrel{\text { def }}{=} \widehat{A}$ is a compact connected locally connected (not arcwise connected) group for which $G / M(G)$ is a circle group. In other words, $M(G / M(G))$ need not be zero in general! One notes that, in the absence of metrizability, this is the starting point of a transfinite ascending recursion process which we shall not pursue in this paper.

However, in view of Theorem 2.13(iv), in the metric case, the hypothesis that $G$ be torus free is frequently no restriction of generality.

Proposition 2.14. Let $\Gamma$ be a compact finite-dimensional abelian group. Then we have the following conclusions:

(i) $\Gamma \cong \mathbb{T}^{m} \times G$ with a unique maximal torus subgroup $\mathbb{T}^{m} \times\{1\}$, and some torus free compact $n$-dimensional group $G, m+n=\operatorname{dim} \Gamma$.

(ii) There is a compact zero-dimensional subgroup $\Delta$ of $G$ and a quotient homomorphism $\phi: \mathbb{R}^{n} \times \Delta \rightarrow G$ with a discrete kernel isomorphic to a lattice $\mathbb{Z}^{p}$ with $p \leq n=\operatorname{dim} G$.

(iii) The arc component of 1 in $G$ is $G_{a}=\exp L(G)=\phi\left(\mathbb{R}^{n} \times\{1\}\right)$. The arc components of $G$ are the sets $d=G_{a}=G_{a} d=\phi\left(\mathbb{R}^{n} \times\{d\}\right)=d \exp L(G)$, and $\phi$ maps the arc component $\mathbb{R}^{n} \times\{d\}$ continuously and bijectively onto $d G_{a}$.

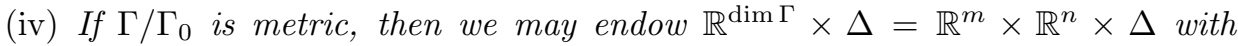
an invariant product metric $d=d_{0} \times d_{1}$ with a Hilbert space metric $d_{0}$ on $\mathbb{R}^{\operatorname{dim} \Gamma}$ and an ultrametric $d_{1}$ on $\Delta$, and give $\Gamma$ the quotient metric $d_{\Gamma}$ for the homomorphism

$$
\begin{gathered}
\Phi: \mathbb{R}^{\operatorname{dim} \Gamma} \times \Delta \rightarrow \Gamma, \\
(X, Y, d) \mapsto\left(X+\mathbb{Z}^{n}, \phi(d, Y)\right): \mathbb{R}^{m} \times \mathbb{R}^{n} \times \Delta \rightarrow \mathbb{T}^{m} \times G \cong \Gamma .
\end{gathered}
$$

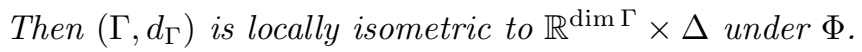

Proof. (i) By Proposition 2.9, the component $\Gamma_{0}$ is metric, hence $M(\Gamma)=M\left(\Gamma_{0}\right)$ is metric. Thus Theorem 2.13(iii) proves $\Gamma \cong M(\Gamma) \times G$ with a torus $M(\Gamma)$ and a torus free compact group $G$. We have $\operatorname{dim} \Gamma=\operatorname{dim} M(\Gamma)+\operatorname{dim} G$. This proves (i).

(ii) From Theorem 2.5 we may identify $L(G)$ with $\mathbb{R}^{n}$, and we get $\phi: L(G) \times \Delta \rightarrow$ $G, \phi(X, d)=d \exp X$, a local isomorphism. (Recall from the proof of Theorem 2.5 that totally disconnected closed subgroups of $\mathbb{R}^{n}$ are discrete lattices.)

(iii) From Corollary 2.4 we know that $\phi$ maps arc components as stated. If $\exp X=1$, then $\exp \mathbb{R} \cdot X$ is a circle group or is trivial. But $G$ does not contain any circle groups. Hence $X=0$ and $\phi$ maps $\mathbb{R}^{n} \times\{1\}$ injectively. Thus (iii) is proved.

(iv) is a straightforward consequence of (i) and (ii).

Let us recall at this point that for a compact connected nonabelian group $G$, the commutator group $G^{\prime}$, which is a closed semisimple nonsingleton compact subgroup, contains a copy of $\mathrm{SO}(3)$ or a copy of $\mathrm{SU}(2)$ and thus is at least 3-dimensional. Hence a compact connected group $G$ with $\operatorname{dim} G \leq 2$ is abelian.

Definition 2.15. A solenoid is a 1-dimensional compact connected group. A proper solenoid is a solenoid which is not a circle group. 
Proposition 2.16. A compact abelian group $G$ is a solenoid iff its character group is isomorphic to a nonzero subgroup of $\mathbb{Q}$. It is proper iff this subgroup is not free.

Proposition 2.17. There is a 2-dimensional compact connected group $G$ which is not a product of two solenoids.

Proof. Pontryagin (see [17, I, Beispiel 15, Seite 48 and II, Beispiel 68, Seite 39]) has exhibited subgroups $A$ of $\mathbb{Q}^{2}$ with $\mathbb{Z}^{2} \subseteq A \subseteq \mathbb{Q}^{2}$ which have no nondegenerate direct summands. For such a group, $G=\widehat{A}$ is a 2-dimensional compact connected group which is not a product of two solenoids.

A continuum is a compact connected space. It is called decomposable if it is the union of two proper subcontinua; otherwise, it is indecomposable. The proper solenoids are known to be indecomposable metric continua. The following theorem shows that this situation cannot occur for compact groups having dimension greater than one. For abelian compact groups, the following theorem was proved by van Heemert [21]; his article also reflects most of the earlier history of these continua. However, even in the case of abelian groups, it is sensible to present a complete proof which reflects the current state of information on compact groups and meets modern demands of completeness and detail.

Theorem 2.18. For a nondegenerate compact group $G$ the following statements are equivalent:

(i) $G$ is a proper solenoid.

(ii) The underlying space of $G$ is an indecomposable continuum.

Proof. We just noted that (i) $\Longrightarrow$ (ii), and observed that the circle is decomposable. Therefore we must prove that $\operatorname{dim} G>1$ implies the decomposability of $G$. By (ii) the group $G$ is connected.

(a) We preface the proof by the following remark. Assume that $N$ is a compact and connected normal subgroup of $G$ and that $p: G \rightarrow G / N$ is the quotient morphism. If $X \subseteq G / N$ is connected, then $p^{-1}(X)$ is connected. Thus, if $G / N$ is decomposable, then $G$ is decomposable.

(b) Assume next that $G$ is semisimple. The structure theory of compact connected semisimple groups says that there is a family of simple compact connected Lie groups $\left\{G_{j}: j \in J\right\}$ and a totally disconnected central subgroup $D$ such that $G \cong\left(\prod_{j \in J} G_{j}\right) / D$ (see, for example, [3, Chap. 9]). Identify $G$ with this factor group. If $G$ is not degenerate, pick an $i \in J$. Then $N \stackrel{\text { def }}{=} \prod_{j \in J-\{i\}} G_{j} D / D$ is a compact connected normal subgroup of $G$ and $G / N \cong G_{i} /\left(N \cap G_{i}\right)$ is a simple connected Lie group, and thus is a compact manifold of dimension at least 3 . Hence it is decomposable. From part (a) of the proof we derive that nondegenerate compact connected semisimple groups are decomposable.

(c) The commutator subgroup $G^{\prime}$ of a compact connected group $G$ is closed and semisimple [3]. We claim that $G$ is abelian. Indeed, assume the contrary. We have $G=Z_{0} G^{\prime}$, where $Z_{0}$ is the identity component of the center, and then $G / Z_{0} \cong G^{\prime} /\left(Z_{0} \cap G^{\prime}\right)$ is semisimple and nonsingleton. Then $G / Z_{0}$ is decomposable by (b), and thus $G$ is decomposable by (a). This conclusion contradicts (ii) and thus proves the claim. We may therefore assume for the remainder of the proof that $G$ is abelian. 
(d) Assume that $G$ is a compact connected abelian group. Then $\widehat{G}$ is a torsion free group whose rank is at least 2. Hence we find a pure subgroup $P$ of rank 2 . (Indeed, let $F$ be a free subgroup of maximal rank. Write it in the form $F_{1} \oplus F_{2}$ with $\operatorname{rank} F_{1}=2$ and set $P=\left\{\chi \in \widehat{G}: n \cdot \chi \in F_{1}\right.$ for some $\left.n \in \mathbb{N}\right\}$.) Let $C=P^{\perp}$, the annihilator of $P$ in $G$. Then $\widehat{C} \cong \widehat{G} / P$ and $\widehat{G / C} \cong P$. Since $P$ is pure, $\widehat{G} / P$ and thus $\widehat{C}$ are torsion free. Hence $C$ is connected. In view of part (a) of the proof, $G$ is decomposable if $G / C$ is decomposable. Furthermore, $\operatorname{rank} P=2 \mathrm{im}$ plies $\operatorname{dim} G / C=2$. In order to complete the proof it therefore suffices to prove that a 2-dimensional compact connected abelian group is decomposable. By Theorem 2.13 (iv) we may assume that $G$ is torus free, since $G$ is clearly decomposable if $G$ contains a circle group as a factor. Using Proposition 2.14, we obtain a compact zero-dimensional subgroup $\triangle$ and a closed $\epsilon$-ball neighborhood $B$ of the origin in $L(G) \cong \mathbb{R}^{2}$ such that $\phi$ maps $\triangle \times B$ homeomorphically onto an identity neighborhood $W$ of $G$. As in Proposition 2.2 and its proof, we set $D=\exp ^{-1}(\triangle)$ and note that $\exp X=\phi(\delta, Y)=\delta \exp Y$ with $\delta \in \triangle$ means $\delta=\exp (X-Y)$, and thus $X=Y+d$ with $d \in D$. Thus, identifying $L(G)$ with $\mathbb{R}^{2}$, we see that $\exp : \mathbb{R}^{2} \rightarrow G$ maps $D+B$ bijectively and continuously onto $W \cap \exp L(G)$. Let $U=\operatorname{Int} B$, the manifold interior of $B$. By Proposition 2.2(ii), the closed subgroup $D$ of $\mathbb{R}^{2}$ is a discrete lattice and therefore is countable. Thus $D+U$ is a countable disjoint union of open disks in the plane. Therefore its complement $E \stackrel{\text { def }}{=} \mathbb{R}^{2}-\{D+U\}$ is connected. By Proposition 2.2(iii), the set $\exp L(G)$ is dense in $G$ and the complement $\exp L(G)-W=\exp E$ is dense in the complement $A \stackrel{\text { def }}{=} G-\phi(\triangle \times U)$. Note that $A$ is a compact subset of $G$ since $\phi$ is an open map, and that $\triangle A=A$ because $\phi(\triangle \times U)$ is stable under the multiplication with $\triangle$. Also, $A$, being the closure of a connected set, is connected. Every arc component of $W$ is of the form $\delta \exp B$, $\delta \in \triangle$, and its intersection with $A$ is $\delta \exp \partial B$.

Let $K_{1}$ be a proper compact open subgroup of $\triangle$, and set $K_{2}=\triangle-K_{1}$. Set $C_{1}=A \cup \phi\left(K_{1} \times B\right)$ and $C_{2}=A \cup \phi\left(K_{2} \times B\right)$. Since each arc component of the compact set $\phi\left(K_{j} \times B\right), j=1,2$, intersects the continuum $A$, each of the sets $C_{1}$ and $C_{2}$ is a continuum. Therefore, since $C_{1}, C_{2}$ are proper subcontinua of $G$ and $G=C_{1} \cup C_{2}$, the space $G$ is decomposable as claimed.

\section{Compact Metrizable Groups and CiPH}

A flow on a space $X$ is a continuous function $\varphi: X \times \mathbb{R} \rightarrow X$ such that $\varphi(x, 0)=x$ and $\varphi(\varphi(x, s), t)=\varphi(x, s+t)$ for all $s, t \in \mathbb{R}$ and $x \in X$. The map $\varphi_{t}: X \rightarrow X$ defined by $\varphi_{t}(x)=\varphi(x, t)$ is a homeomorphism since $\varphi_{-t}=\varphi_{t}^{-1}$. A point $p \in X$ is an invariant point of $\varphi$ if $\varphi_{t}(p)=p$ for all $t \in \mathbb{R}$, and the $\operatorname{set} \bigcap\left\{\operatorname{fix}\left(\varphi_{t}\right): t \in \mathbb{R}\right\}$, called the invariant set of the flow, is a closed subset of $X$ if $X$ is a Hausdorff space.

Definition 3.1. A flow $\varphi: M \times \mathbb{R} \rightarrow M$ on a metric space $(M, d)$ is called uniform if it satisfies the following conditions:

(i) $d(x, \varphi(x, t)) \leq C|t|$ for some positive $C$ and all $x \in M, t \in \mathbb{R}$.

(ii) There is a real number $p \geq 0$ such that for all $x \in M$ and $t \in \mathbb{R}, \varphi(x, t)=x$ iff $t \in p \mathbb{Z}$.

Proposition 3.2. Let $(M, d)$ be a compact metric space with a uniform flow $\varphi$. Then every nonempty closed subset of $M$ is the fixed point set of an orbit-preserving autohomeomorphism of $M$. In particular, $M$ has $\mathrm{CIPH}$. 
Proof. In the case where $p>0$ it suffices to consider $p=1$. The formulation here is a matter of technical convenience for later proofs. If 3.1(ii) is satisfied with $p>0$, define a new metric $D$ and a new flow $\psi$ by $D(x, y)=(p C)^{-1} d(x, y)$ and $\psi(x, t)=\varphi(x, p t)$. Then

$$
D(x, \psi(x, t))=(p C)^{-1} d(x, \varphi(x, p t)) \leq(p C)^{-1} \cdot C|p t|=|t|
$$

and $\psi$ satisfies 3.1(ii). When $p=1$ there is a free circle action on $M$ and the proposition follows from $[13,2.3]$.

Now suppose we have the case corresponding to $p=0$ and $A$ is a nonempty closed subset of $M$. Let $r(x)=\frac{1}{2 C} d(x, A)$ for $x \in M$ and define a mapping $h: M \rightarrow M$ by $h(x)=\varphi(x, r(x))$ for $x \in M$. Clearly, $\operatorname{fix}(h)=A$.

To see that $h$ is one-to-one, suppose that $h(x)=h(y)$. Then $x$ and $y$ must lie in the same orbit and, for some real number $t, y=\varphi(x, t)$. Then

$$
\varphi(x, r(x))=\varphi(\varphi(x, t), r(y))=\varphi(x, t+r(y)) .
$$

Thus $t=r(x)-r(y)$. The triangle inequality applied to $x, y, A$ implies

$$
d(x, y) \geq|d(x, A)-d(y, A)| .
$$

Since $C|t| \geq d(x, y)$, we have $C|t| \geq 2 C|r(x)-r(y)|$. Therefore $t=0$ and $r(x)=$ $r(y)$. Then we have

$$
\varphi(\varphi(x, r(x)),-r(x))=\varphi(\varphi(y, r(y)),-r(y)) .
$$

Consequently, $x=\varphi(x, 0)=\varphi(y, 0)=y$, and $h$ is one-to-one.

To complete the proof that $M$ has CIPH, it suffices to show that $h$ is onto. To see this, let $y \in M$. For some $b \in \mathbb{R}$, the metric $d$ is bounded by $b$. Let $x$ denote the unique point in the orbit $\varphi(\{y\} \times \mathbb{R})$ such that $\varphi(x, b)=y$ and let $[x, y]$ denote the arc in $\varphi(\{y\} \times \mathbb{R})$ with endpoints $x$ and $y$. Since $h \mid[x, y]$ is an order-preserving homeomorphism from $[x, y]$ onto $h([x, y])$ such that $h(x)<y$, and $h(y) \geq y$, it follows that $y \in h([x, y])$. Consequently, $h$ is onto as required.

Lemma 3.3. If $\left(X_{j}, d_{j}\right), j=1,2$, are metric spaces and $\varphi$ is a uniform flow on $\left(X_{2}, d_{2}\right)$, then $X=X_{1} \times X_{2}$ is a metric space with respect to the metric $D$ defined by

$$
D\left(\left(x_{1}, x_{2}\right),\left(x_{1}^{\prime}, x_{2}^{\prime}\right)\right)=\max \left\{d_{1}\left(x_{1}, x_{1}^{\prime}\right), d_{2}\left(x_{2}, x_{2}^{\prime}\right)\right\},
$$

and the flow $\Phi$ on $X$ given by $\Phi\left(\left(x_{1}, x_{2}\right), t\right)=\left(x_{1}, \varphi\left(x_{2}, t\right)\right)$ is uniform on $(X, D)$.

Proof. Note that

$$
D\left(\left(x_{1}, x_{2}\right), \Phi\left(\left(x_{1}, x_{2}\right), t\right)=D\left(\left(x_{1}, x_{2}\right),\left(x_{1}, \varphi\left(x_{2}, t\right)\right)=d_{2}\left(x_{2}, \varphi\left(x_{2}, t\right)\right) .\right.\right.
$$

Condition 3.1(i) follows readily, and 3.1(ii) is straightforward.

Proposition 3.4. If $(M, d)$ is a compact metric space with a uniform flow and $X$ is a metrizable space, then $M \times X$ has $\mathrm{CIPH}$.

Proof. By Lemma 3.3 there is a metric $\rho$ for $M \times X$ and a flow $\varphi$ which is uniform on $(M \times X, \rho)$. The proof of Proposition 3.2 can be applied to $M \times X$. Since $M \times X$ need not be compact, the one adjustment required is to use the compactness of $M$ to show that the mapping $h$ is a closed mapping and hence an autohomeomorphism of $M \times X$. 
Recall that each compact metrizable group and each abelian metrizable group $G$ has a metric $d$ satisfying $d(g x h, g y h)=d(x, y)$. Such metrics will be called invariant. They are completely characterized by the function $g \mapsto\|g\|: G \rightarrow \mathbb{R}$ defined by $\|g\|=d(1, g)$, from which $d$ is recoverable by $d(g, h)=\left\|g^{-1} h\right\|$. The function $\|\cdot\|$ satisfies $\left\|x^{-1}\right\|=\|x\|,\|g h\| \leq\|g\|+\|h\|,\left\|h g h^{-1}\right\|=\|h\|$ and $\|g\|=0$ iff $g=1$. We call a function $\|\cdot\|$ an invariant norm on $G$ and remember that the invariant norms are in bijective correspondence with the invariant metrics on a group. (See e.g. [4, Chap. IX, p. TG IX.24] where this is discussed for abelian groups; the arguments work for invariant metrics quite generally.)

Lemma 3.5. Assume that $G$ is a group with an invariant norm $\|\cdot\|$ and there is a one-parameter subgroup $\alpha: \mathbb{R} \rightarrow G$ such that $\|\alpha(t)\| \leq C|t|$ for some positive number $C$. Suppose that $N$ is a closed normal subgroup of $G$ not containing $\alpha(\mathbb{R})$. Then there is a metric $D$ on $G / N$ such that the flow $(N g, t) \mapsto N g \alpha(t)$ is uniform on $(G / N, D)$.

Proof. We define $\|N g\|_{N}=\inf \{\|n g\|: n \in N\}$. Then $\|\cdot\|_{N}$ is an invariant norm on $G / N$. The topology defined by the metric $D$ associated with this norm is the quotient topology.

We must verify (i) and (ii) of Definition 3.1 for the flow $\varphi$ on $G / N$ defined by $\varphi(N g, t)=N g \alpha(t)$. Regarding (i), we compute

$$
\begin{aligned}
D(N g, \varphi(N g, t)) & =D(N g, N g \alpha(t))=\|N \alpha(t)\|_{N} \\
& =\inf \{\|n \alpha(t)\|: n \in N\} \leq\|\alpha(t)\| \leq C(t)
\end{aligned}
$$

by hypothesis on $\alpha$. Thus 3.1(i) is satisfied.

Regarding (ii), we note that $\varphi(N g, t)=N g$ means $g N \alpha(t)=N g \alpha(t)=N g=$ $g N$, i.e., $\alpha(t) \in N$. Since $\alpha(\mathbb{R})$ is not contained in $N$, the inverse image $\alpha^{-1}(N)$ is a closed proper subgroup of $\mathbb{R}$ and thus is of the form $p \mathbb{Z}$ with a nonnegative $p \in \mathbb{R}$. If $p=0$, then $\varphi(N g, t)=H g$ holds iff $t=0$. If $p$ is positive, then $\varphi(N g, t)=H g$ holds iff $t \in p \mathbb{Z}$. Thus 3.1(ii) holds, and the proof is complete.

Proposition 3.6. The Cantor set and the space of irrationals have CIPH.

Proof. Note that the space of irrationals is homeomorphic to $\mathbb{Z}^{\omega}$ ([1]) and the Cantor set is homeomorphic to $\mathbb{D}^{\omega}$, where $\mathbb{D}$ denotes the two-point discrete group ([5]). We shall only deal here with the Cantor set.

Fix a metric $d$ for $\mathbb{D}^{\omega}$ and let $A$ be a nonempty closed subset of $\mathbb{D}^{\omega}$. Then the complement $\mathbb{D}^{\omega}-A$, as an open subspace of $\mathbb{D}^{\omega}$, can be written as a countable (finite or infinite) disjoint union $C_{1} \cup C_{2} \cup \ldots$ of closed and open subspaces of $\mathbb{D}^{\omega}$ each of which is homeomorphic to $\mathbb{D}^{\omega}$. Since translation by a nonzero element in an abelian group determines an autohomeomorphism of the group, it follows that there is a sequence of fixed point free autohomeomorphisms $h_{1}, h_{2}, \ldots$ of $C_{1}, C_{2}, \ldots$ respectively. Moreover, we can choose each $h_{i}$ so that $d\left(h_{i}(x), x\right)<\frac{1}{i}$ for $x \in C_{i}$. It only remains to observe that the function $h: \mathbb{D}^{\omega} \rightarrow \mathbb{D}^{\omega}$ defined by

$$
h(x)= \begin{cases}x & \text { if } x \in A, \\ h_{i}(x) & \text { if } x \in C_{i},\end{cases}
$$

is an autohomeomorphism of $\mathbb{D}^{\omega}$ with $\operatorname{fix}(h)=A$.

Lemma 3.7. Every compact metrizable totally disconnected infinite group is homeomorphic to the Cantor set and hence has $\mathrm{CIPH}$. 
Proof. The proof follows from [5] and Proposition 3.6.

Theorem 3.8. In every infinite compact metrizable group $G$ each nonempty closed subset is the fixed point set of some autohomeomorphism. If $G$ is not totally disconnected, then $G$ admits a metric $d$ and a uniform flow on $(G, d)$ defined by the action of some one-parameter group of $G$ on the right. In particular, every nonempty closed subset of $G$ is the fixed point set of an orbit-preserving autohomeomorphism of $G$.

Proof. By Mostert's Cross Section Theorem [11, 1.14], every compact group $G$ possesses a zero-dimensional compact subset $Z$ homeomorphic to $G / G_{0}$ such that $(z, g) \longmapsto z g: Z \times G_{0} \rightarrow G$ is a homeomorphism. Since $G$ is infinite, at least one of $G / G_{0}$ or $G_{0}$ is infinite. If $G_{0}$ is not degenerate, in view of Lemma 3.3 it suffices to prove the theorem for $G_{0}$. If $G_{0}$ is singleton, then $G$ is totally disconnected and the assertion follows from Lemma 3.7.

Thus the proof will be completed if we establish the claim for $G$ connected, which we shall assume for the remainder of the proof. Since every compact group is the projective limit of Lie groups, there is a closed normal subgroup $M$ such that $G / M$ is a Lie group. (See e.g. [3, Chap. IX, p. LIE IX.99, Corollaire 1].) The identity component $K$ of $M$ is a compact connected normal subgroup of $G$. There exists a compact connected normal subgroup $H$ of $G$ such that $K \cap H$ is zero-dimensional and central (see e.g. [11, p. 299, 2.5]). Since $H /(K \cap H) \cong G / M$, the group $H$ is finite-dimensional. The map $q: K \times H \rightarrow G, q(k, h)=k h$ is a surjective continuous morphism. Thus $G \cong(K \times H) / N$ with $N=\operatorname{ker} q$. In view of Lemma 3.5 it suffices to prove the required data on $K \times H$. Then by Lemma 3.3 it suffices again to prove the claim for $H$. Thus we may assume now that $G$ is compact connected and finite-dimensional. Now $G$ is the semidirect product $G^{\prime} \times A$ of the closed normal commutator subgroup $G^{\prime}$ and an abelian subgroup $A$ isomorphic to $G / G^{\prime}$ (see [10]). Since $G^{\prime}$ is finite-dimensional and semisimple, it is a Lie group. By Lemma 3.3 it suffices to prove the assertion for each of the two factors separately.

Case 1. $G$ is a semisimple Lie group. We claim that the theorem holds for every compact connected Lie group $G$. Every such $G$ contains a maximal torus (see [3, p. LIE IX.8]) and has an invariant Riemannian metric $d$ which induces on $T$ an invariant Riemannian metric and thus induces on each circle group in $T$ an invariant Riemannian metric. However, an invariant Riemannian metric on $\mathbb{R} / \mathbb{Z}$ is a positive multiple of the metric given by $d(s+\mathbb{Z}, t+\mathbb{Z})=|s-t|$. Thus we find a one-parameter subgroup $\alpha: \mathbb{R} \rightarrow G$ such that $\|\alpha(t)\| \leq C|t|$ with the invariant norm associated with $d$. Then Lemma 3.5 yields a proof of the assertion in this case with $N=\{1\}$.

Case 2. $G$ is a finite-dimensional abelian group. Then $G=T \times A$ with a unique maximal torus and a torus free factor, by Theorem 2.13(iv). By Lemma 3.3 the proof will be accomplished if it can be done for each of the factors separately. In the proof of Case 1 we took care of the case of a Lie group. Thus there remains only the case that $G$ is a torus free compact connected abelian group.

Now let $\beta: \mathbb{R} \rightarrow G$ denote any nondegenerate one-parameter subgroup. One exists since $G$ is assumed to be nondegenerate. Since $G$ is torus free, we have

$$
\beta(t)=0 \text { iff } t=0 .
$$

In particular, $\beta(1) \neq 0$. The characters of $G$ separate the points; hence there is a character $\chi: G \rightarrow \mathbb{T}=\mathbb{R} / \mathbb{Z}$ such that $\chi(\beta(1)) \neq 0$. The morphism $\chi \circ \beta: \mathbb{R} \rightarrow$ 
$\mathbb{R} / \mathbb{Z}$ lifts to a morphism $\lambda: \mathbb{R} \rightarrow \mathbb{R}$ such that $\chi \circ \beta=p \circ \lambda$ with the quotient (covering) morphism $p: \mathbb{R} \rightarrow \mathbb{R} / \mathbb{Z}$. (This is elementary; it also follows from a topological fact since $\mathbb{R}$ is simply connected and $p$ is a covering map.) Every continuous endomorphism $\lambda$ of $\mathbb{R}$ is of the form $\lambda(t)=r t$ for some $r \in \mathbb{R}$. Since $p(r)=\chi \circ \beta(1) \neq 0$, we certainly have $r \neq 0$. We then have $\chi(\beta(t))=r t+\mathbb{Z}$, and define $\alpha: \mathbb{R} \rightarrow G$ by $\alpha(t)=\beta\left(\frac{t}{r}\right)$. Then (3.1) implies

$$
\alpha(t)=0 \text { iff } t=0
$$

and we have

$$
\chi \circ \alpha=p: \mathbb{R} \rightarrow \mathbb{T} .
$$

Now we set $K=\operatorname{ker} \chi$ and define

$$
\Psi: K \times \mathbb{R} \rightarrow G, \quad \Psi(k, t)=k+\alpha(t) .
$$

Claim: $\Psi$ is surjective. For a proof let $g \in G$. Pick $t \in \mathbb{R}$ such that $\chi(g)=t+\mathbb{Z}$ and set $k=g-\alpha(t)$. Then $\chi(g-\alpha(t))=\chi(g)-\chi(\alpha(t))=\chi(g)-(t+\mathbb{Z})=0$ in view of (3.3). Hence $k \in K$ and thus $g=k+\alpha(t)=\Psi(k, t)$. This proves the claim.

Now Lemma 3.5 shows that it is sufficient to prove the claim for $K \times \mathbb{R}$. Then by Lemma 3.3 it suffices to prove the claim for $\mathbb{R}$ which is obvious.

Corollary 3.9. If $G$ is a positive-dimensional compact metrizable group and $X$ is a metrizable space, then $G \times X$ has $\mathrm{CIPH}$.

Proof. The proof is immediate by Theorem 3.8 and Proposition 3.4.

Corollary 3.10. A compact metrizable group has CIPH iff it is either degenerate or infinite.

Proof. An autohomeomorphism of a nondegenerate finite discrete group cannot fix all points but one.

Corollary 3.11. Every compact metrizable group has CIP.

Proof. Every nonempty subset of a finite discrete group is the fixed point set of a retraction.

\section{Some Consequences and Ramifications}

In [14, p. 134] a space $Y$ is defined to have property $W$ (strong) if it admits a homotopy $H: Y \times[0,1] \rightarrow Y$ such that $H(y, t)=y$ iff $t=0$. We note that a metric space has property $W$ (strong) if it admits a uniform flow, since then we can set $H(x, t)=\varphi(x, t)\left(\right.$ or $\varphi\left(x, \frac{t}{2 p}\right)$ for $\left.p>0\right)$. It is shown in $[16,3.14]$ that if $M$ is a connected metrizable space with property $W$ (strong) and $X$ is a zero-dimensional compact (Hausdorff) space, then $M \times X \times X$ has CIP iff $X$ is metrizable. In the next result we use these facts and the concept of CIPH to give a characterization of the compact zero-dimensional spaces which are metrizable.

Proposition 4.1. If $(M, d)$ is a compact connected metric space admitting a uniform flow and $X$ is a compact zero-dimensional space, then $M \times X \times X$ has $\mathrm{CIPH}$ iff $X$ is metrizable. 
Proof. Sufficiency. If $X$ is metrizable, then $X \times X$ is metrizable and $M \times X \times X$ has CIPH by Proposition 3.4.

Necessity. Since a space having CIPH has CIP, the necessity follows from the remarks preceding the proposition.

We note that if $X$ is a nondegenerate compact zero-dimensional metrizable space, then $X^{\lambda} \times X^{\lambda}$ is metrizable iff $X^{\lambda}$ is metrizable iff $\lambda$ is a countable cardinal. Moreover, when $\lambda$ is uncountable we have $X^{\lambda} \times X^{\lambda}=X^{\lambda}$. Consequently, we can apply Proposition 4.1 to obtain the following result.

Corollary 4.2. If $(M, d)$ is a compact connected metric space admitting a uniform flow and $X$ is a nondegenerate compact zero-dimensional metrizable space, then $M \times X^{\lambda}$ has $\mathrm{CIPH}$ iff $\lambda$ is a countable cardinal.

We note that if a Hausdorff space $D \times M$ has CIPH, where $D$ is a totally disconnected space and $M$ is a connected space, then $M$ has CIPH since, given a nonempty closed subset $A$ of $M$, an autohomeomorphism $h$ of $D \times M$ with fix $(h)=$ $D \times A$ must preserve components and thereby determine an autohomeomorphism of $M$ whose fixed point set is $A$. Since an $n$-manifold having a compact boundary component with a nonzero Euler characteristic does not have CIPH $([13,3.1])$, it follows that $\mathbb{D}^{\omega} \times I^{\lambda}, I=[0,1]$, does not have CIPH if $\lambda$ is an odd integer. If $G$ is a compact metrizable group which is not totally disconnected, then Corollary 3.9 shows that $G \times I^{\lambda}$ has CIPH if $\lambda$ is a countable cardinal. However, no nonmetrizable ANR-compactum has CIP ([6, 3.4]). Since a compact Lie group is an ANR-space, its product with a Tychonov cube is a nonmetrizable ANR-compactum and we obtain the following result.

Proposition 4.3. If $G$ is a compact Lie group, then $G \times I^{\lambda}$ has CIPH iff $\lambda$ is a countable cardinal.

Remark 4.4. We note that Corollary 4.2 and Proposition 4.3 provide examples which contrast the metrizable and nonmetrizable cases regarding CIPH. In what follows, let $\lambda$ be an uncountable cardinal.

1. Let $G$ be a compact Lie group and consider the Hilbert cube $Q=I^{\omega}$. By Proposition 4.3, $G \times I^{\lambda}=G \times Q^{\lambda}$ does not have CIPH. However, since $G$ has CIPH by Theorem 3.8 and $Q$ has CIPH by [13, 12.4], $G \times Q^{\lambda}$ is a product of homogeneous spaces each of which has CIPH. This is in contrast to the metric case, where it is not known if every homogeneous locally contractible space has CIPH.

2. Let $G$ be a compact connected metrizable group and let $C=\mathbb{D}^{\omega}$. Then $G \times \mathbb{D}^{\lambda}=G \times C^{\lambda}$ does not have CIPH by Theorem 3.8 and Corollary 4.2. By Theorem 3.8, $G \times C^{\lambda}$ is a product of compact groups each of which has CIPH. This is in contrast to the metric case since, by Corollary 3.10, a metrizable product of compact groups has CIPH iff it is either degenerate or infinite.

We remark also that the hypothesis of compactness in Proposition 3.2 can be relaxed so that the conclusion of the proposition is valid for free $\mathbb{R}$-actions. However, one cannot conclude that connected metrizable groups must have CIP, since there exists a connected subgroup of the plane $\mathbb{R}^{2}$ which fails to have CIP $[16,2.2]$.

Finally, we end this section by noting that any metrizable space $M$ admitting a flow satisfying condition (ii) of Definition 3.1 admits distinct flow structures corresponding to the closed subsets of $M$. 
Proposition 4.5. Let $M$ be a metrizable space admitting a flow satisfying condition (ii) of Definition 3.1. If $A$ is a closed subset of $M$, then there is a flow on $M$ whose invariant set is $A$.

Proof. In [2] it is shown that every closed subset of a metrizable space $X$ is the invariant set of a flow on $X$ iff $X$ admits a flow having an empty invariant set. The proof follows, since a flow $\varphi$ on $M$ satisfying condition (ii) of Definition 3.1 has no invariant points.

By Lemma 3.3 a product of two metrizable spaces will admit a uniform flow if one of the factor spaces does. Thus we obtain the following result.

Corollary 4.6. Let $M$ be a metrizable space and let $G$ be a compact metrizable positive-dimensional group. Then every closed subset of $G \times M$ is the invariant set of some flow on $G \times M$.

\section{ACKNOWLEDGEMENTS}

We thank the referee for many helpful remarks and suggestions which eliminated typographical errors and led to a number of improvements, notably in the historical record and our references. The results of the article in the meantime also appear in A. Chigogidze, Inverse Spectra, North-Holland, Amsterdam, 1996, and are used in [7] to show that any uncountable product of a metrizable compact group has $\mathrm{CIPH}$ and that homogeneous metrizable continua need not have CIPH. A part of the information will also appear in K. H. Hofmann and S. A. Morris, The Structure of Compact Groups, de Gruyter, Berlin, 1997.

\section{REFERENCES}

1. P. S. Alexandrov and P. S. Urysohn, Über nulldimensionale Mengen, Math. Ann. 98 (1928), 89-106.

2. A. Beck, On invariant sets, Ann. of Math. 67 (1958), 99-103. MR 19:1064c

3. N. Bourbaki, Groupes et algèbres de Lie, Chap. IX, Masson, Paris, 1982. MR 84:22001

4. N. Bourbaki, Topologie générale, Herman, Paris, 1974. MR 30:3439 (earlier ed.)

5. L. E. J. Brouwer, On the structure of perfect sets of points, Proc. Akad. Amsterdam 12 (1910), $785-794$.

6. A. Chigogidze and J. R. Martin, Fixed point sets of Tychonov cubes, Topology Appl. 64 (1995), 201-218. MR 96f:54049

7. to appear.

8. J. Dixmier, Quelques propriétés des groupes abéliens localement compacts, Bull. Sci. Math. (2) 81 (1957), 38-48. MR 20:3196

9. T. Fay, E. Oxford, and G. Wells, Preradicals in abelian groups, Houston J. Math. 8 (1982), 39-52. MR 83m:20074

10. K. H. Hofmann, Sur la décomposition semidirecte de groupes compacts connexes, Symposia Mathematica 26 (1975), 471-476. MR 53:5805

11. K. H. Hofmann and P. S. Mostert, Elements of Compact Semigroups, Charles E. Merill Books, Columbus, OH, 1966. MR 35:285

12. Y. Iwamoto, Fixed point sets of transformation groups of Menger manifolds, their pseudointeriors and their pseudo-boundaries, Topology Appl. 68 (1990), 267-283. MR 97a:54035

13. J. R. Martin, Fixed point sets of homeomorphisms of metric products, Proc. Amer. Math. Soc. 103 (1988), 1293-1298. MR 89h:55003

14. J. R. Martin, L. G. Oversteegen and E.D.Tymchatyn, Fixed point sets of products and cones, Pacific J. Math. 101 (1982), 133-139. MR 83j:54025

15. J. R. Martin and S. B. Nadler, Examples and questions in the theory of fixed point sets, Can. J. Math. 31 (1979), 1017-1032. MR 80m:54059 
16. J. R. Martin and W. A. R. Weiss, Fixed point sets of metric and nonmetric spaces, Trans. Amer. Math. Soc. 284 (1984), 337-353. MR 85h:54085

17. L. S. Pontryagin, Topologische Gruppen I und II, Teubner Leipzig (German translation 1959, 1958 of the second Russian edition). MR 19:152e; MR 20:3925

18. H. Schirmer, On fixed point sets of homeomorphisms of the n-ball, Israel J. Math. 7 (1969), 46-50. MR 39:7590

19. 373-378. MR 45:7699

20. _ Fixed point sets of continuous selfmaps, Fixed Point Theory Proc. (Sherbrooke, 1980), Lecture Notes in Math. 866 (1981), 417-428. MR 84h:54045

21. A. van Heemert, Toplogische Gruppen und unzerlegbare Kontinua, Comp. Math. 5 (1938), 319-326.

22. N. Vedenissoff, Remarks on the dimension of topological spaces (Russian), Moskov. Gos. Univ. Uč. Zap. 30 (1939), 131-140. MR 2:69i

23. L. E. Ward, Jr., Fixed point sets, Pacific J. Math. 47 (1973), 553-565. MR 51:4205

Department of Mathematics and Statistics, University of Saskatchewan, McLean Hall, 106 Wiggins Road, Saskatoon, SK, S7N 5E6, Canada

E-mail address: chigogid@math.usask.ca

Fachbereich Mathematik, Technische Hochschule, Schlossgartenstrasse 7, D-64289 DARMSTADt, Germany

E-mail address: hofmann@mathematik.th-darmstadt.de

Department of Mathematics and Statistics, University of Saskatchewan, McLean Hall, 106 Wiggins Road, Saskatoon, SK, S7N 5E6, Canada

E-mail address: math@sask.usask.ca 